\title{
Reduced Opioid Use and Reduced Time in the Postanesthesia Care Unit Following Preoperative Administration of Sublingual Sufentanil in an Ambulatory Surgery Setting
}

\author{
Christian D Tvetenstrand, $M D^{1,2^{*}}$ and Michael E Wolff, $M D^{2}$ \\ ${ }^{1}$ Southern Tier Surgical Clinic, Johnson City, NY, USA \\ ${ }^{2}$ United Health Services, Johnson City, NY, USA
}

\begin{abstract}
Background: As the number of surgeries performed in an outpatient setting continues to rise, it is important to continually optimize perioperative pain management to enhance patient outcomes and optimize efficiency of the postanesthesia care unit (PACU). While intravenous (IV) fentanyl is commonly used to provide pain relief both intra- and postoperatively, IV bolus dosing can have negative hemodynamic effects during surgery and induce nausea and vomiting in the PACU. In this study, we evaluated whether preoperative administration of a sublingual sufentanil tablet, a novel analgesic with unique pharmacokinetic characteristics, could reduce overall opioid use and time in the PACU.

Methods: In this prospective medication use evaluation study, a single sublingual sufentanil tablet (SST) 30 mcg was administered prior to surgery in patients undergoing an outpatient procedure. Perioperative opioid use, vasopressor use, and time in the PACU were compared with historical (non-SST-treated) controls.

Results: A total of 127 patients were evaluated with nearly identical baseline demographics. Significantly fewer SSTtreated patients required intraoperative IV opioid following dosing with SST compared with the control group (61.7\% vs. $97.5 \%$, respectively; $p<0.001)$. SST patients received a total preoperative and intraoperative mean opioid dose of $10.9 \mathrm{mg}$ milligram morphine equivalents (MME), while the mean dose for controls was $20.0 \mathrm{mg} \mathrm{MME}(\mathrm{p}<0.001)$. Fewer SST-treated patients required any postoperative opioid $(10.5 \%$ vs. $63.0 \% ; p<0.001)$ with overall opioid utilization being reduced by over $50 \%$ with SST use throughout the perioperative setting (11.8 MME vs. 24.6 MME; $p<0.001)$. Finally, significantly fewer patients in the SST group received adrenergic agonists during surgery and discharge from the PACU occurred $34 \%$ faster than controls ( $36.3 \mathrm{~min}$ vs. $54.9 \mathrm{~min} ; \mathrm{p}<0.001$ ).
\end{abstract}

Conclusion: Preoperative administration of SST results in significant reductions in opioid use during outpatient surgery and facilitates shorter PACU stays.

\section{Keywords}

Sublingual sufentanil, Ambulatory surgery, Opioid sparing, PACU time, Stewardship

\section{Introduction}

Quality pain relief during and after surgery is critical as it contributes to reduced adrenal and physiologic stress on the patient as well as impacts patient satisfaction, time of discharge, readmission rates, and patient care costs [1,2]. This is especially the case for outpatient or ambulatory surgery, where due to the brevity of the procedures and volume of cases, recovery time after surgery is nearly as important as pain relief with regards to successfully discharging the patient to accommodate additional patients in the recovery unit.

Pain during ambulatory surgery is typically managed with IV opioids, namely fentanyl, hydromorphone, and morphine.
Since IV fentanyl is rapidly effective and has no active metabolites, it is one of the most commonly used opioids during

*Corresponding author: Christian D Tvetenstrand, MD, Southern Tier Surgical Clinic, 30 Harrison St, Suite 320, Johnson City, NY 13790, USA, Tel: 607-763-8205, Fax: 607-763-8205

Accepted: August 08, 2020

Published online: August 10, 2020

Citation: Tvetenstrand CD, Wolff ME (2020) Reduced Opioid Use and Reduced Time in the Postanesthesia Care Unit Following Preoperative Administration of Sublingual Sufentanil in an Ambulatory Surgery Setting. J Clin Anesth Pain Manag 4(2):123-128 
Citation: Tvetenstrand CD, Wolff ME (2020) Reduced Opioid Use and Reduced Time in the Postanesthesia Care Unit Following Preoperative Administration of Sublingual Sufentanil in an Ambulatory Surgery Setting. J Clin Anesth Pain Manag 4(2):123-128

and after ambulatory surgery [3,4]. Rapid high peak fentanyl blood concentrations from the initial IV bolus administration during anesthetic induction can contribute to bradycardia and hypotension, which often requires the administration of ephedrine or other adrenergic agents [5]. This peak is followed by a rapid decline in plasma concentrations due to the short ( $<2 \mathrm{~min}$ ) alpha-distribution phase of the drug, and usually a need to re-dose the patient multiple times during the procedure [6]. This rapid peak-trough pharmacokinetic profile with bolus IV administration can be challenging to manage in the intraoperative setting. This adverse hemodynamic effect can also be observed following IV bolus administration of hydromorphone and morphine [7]. Furthermore, when patients awaken from surgery after being treated with repeated dosing of IV opioids intraoperatively, they commonly experience sedation, nausea, and vomiting which can delay recovery [8].

When patients require additional IV opioids in the postanesthesia care unit (PACU) to treat moderate-to-severe pain, further delays in discharge can occur $[9,10]$. Additional nursing time to obtain, dose, and document the IV opioid, additional monitoring time that is required following dosing, and opioid-induced side effects can all result in prolonged PACU stays that can extend the PACU recovery period. This creates additional challenges for the facility since the PACU often becomes the bottleneck in an ambulatory surgery setting. The PACU typically has a limited number of beds and requires a high nurse-to-patient ratio to closely monitor patients emerging from anesthesia, which ultimately makes long stays in the PACU expensive for the facility [11]. Thus, an alternative to IV opioid-based pain management that could minimize overall opioid use and shorten PACU times would be highly desirable.

Sufentanil $30 \mathrm{mcg}$ sublingual tablet (SST; DSUVIA ${ }^{\circ}$, AcelRx Pharmaceuticals, Inc, Redwood City, CA) is approved for use in adults in a certified medically supervised healthcare setting, such as hospitals, surgical centers, and emergency departments, for the management of acute pain severe enough to require an opioid analgesic and for which alternative treatments are inadequate. Sufentanil is more than 1000 times as lipophilic as morphine [12], which allows for rapid transmucosal absorption from sublingual tissues. However, sublingual drug absorption still occurs more slowly than IV administration, as evidenced by SST dosing resulting in a 17fold decreased peak plasma concentration and longer plasma half-time than IV sufentanil administration of the same $30 \mathrm{mcg}$ dose [13]. Clinical studies have shown that effective pain relief occurs within 15 minutes and is sustained for up to 3 hours, in contrast to the rapid plasma fluctuations of IV fentanyl bolus administration [14]. Importantly, the sublingual route blunts the peak plasma concentrations that are normally associated with an adverse hemodynamic effect. Due to SST's unique pharmacokinetic and pharmacodynamic profile, we anticipated that administration of SST prior to ambulatory surgery may reduce IV opioid use during and after surgery, reduce the need for adrenergic agents following anesthetic induction, and ultimately reduce time in the PACU.

\section{Materials and Methods}

This was a prospective medication use evaluation study that assessed the use of preoperative SST in an ambulatory surgery setting. In the prospective group, the study drug SST $30 \mathrm{mcg}$ was to be administered sublingually in the preoperative holding area to all patients approximately 30 minutes prior to incision to allow time for plasma concentrations to be above the published analgesic threshold of sufentanil of 24 $\mathrm{pg} / \mathrm{mL}$ [13]. The control population consisted of patients who underwent the same types of surgery, by the same surgeon, three months immediately prior to the start of the prospective phase. In this control group, IV bolus opioid administration preoperatively and/or intraoperatively was utilized. Control patients' data were gathered from October 2019 - December 2019 and the prospective phase occurred from January 2020 - March 2020. All surgeries, both in the prospective and control groups, were performed at the Universal Health Services Wilson Medical Center in Johnson City, NY.

Patients were eligible for enrollment in the study if they were undergoing ambulatory surgery, defined as an anticipated discharge on the same day as the admission, and were 18 years of age or older. For this analysis, the same exclusion criteria were applied to both populations: Patients were excluded if there were unanticipated complications during the surgery which resulted in an overnight stay.

Data for analysis was obtained from electronic medical records. Primary assessments included opioid requirements and postoperative recovery time. Intraoperative and postoperative opioid use is reported as percent of patients requiring opioids as well as morphine milligram equivalents (MME), calculated using the Practical Pain Management calculator [15]. Time from admission to discharge from the phase 1 unit in the PACU was used to assess postoperative recovery time. Secondary assessments included the intraoperative use of adrenergic agonists (e.g., ephedrine, norepinephrine) and the use of naloxone for respiratory depression. Respiratory adverse events and any unexpected adverse reactions were noted by the hospital staff.

The program was approved by the United Health Services Hospitals Institutional Review Board (IRB\#:IRB00003573; Johnson City, NY). All patients in the prospective group receiving SST provided informed consent. Comparisons between PACU time and opioid use were evaluated using a two-tailed Student's T-Test with an assumed unequal variance. Other comparisons were performed via Chi Square Test.

\section{Results}

A total of 47 patients were enrolled in the prospective SST-treated group and 80 patients were included in the retrospective control population. In both groups, more than $75 \%$ of surgeries were abdominal in nature (e.g., cholecystecto$\mathrm{my}$, hernia repair). Patients in both groups were found to be similar with respect to mean age, body-mass index (BMI), and length of surgery time (Table 1 ). In addition, the ranges of these demographic measurements were also found to be similar for both control and SST-treated patients, encompassing a wide range of ages (up to 90 years) and BMIs (up to 61.4 $\mathrm{kg} / \mathrm{m}^{2}$ ).

For the SST-treated patients, the mean time between 
Citation: Tvetenstrand CD, Wolff ME (2020) Reduced Opioid Use and Reduced Time in the Postanesthesia Care Unit Following Preoperative Administration of Sublingual Sufentanil in an Ambulatory Surgery Setting. J Clin Anesth Pain Manag 4(2):123-128

Table 1: Patient demographics ${ }^{\mathrm{a}}$

\begin{tabular}{|l|l|l|}
\hline Demographic Comparison & Control & SST \\
\hline Mean Age, years & 52.1 & 54.2 \\
(Range) & $(22-90, \mathrm{n}=80)$ & $(18-86, \mathrm{n}=47)$ \\
\hline Mean BMI, kg/m & 32.7 & 32.2 \\
(Range) & $(16.3-59.8, \mathrm{n}=80)$ & $(15.3-61.4, \mathrm{n}=45)$ \\
\hline Mean Length of Surgery, minutes & 40.7 & 37.3 \\
(Range) & $(11-131, \mathrm{n}=78)$ & $(12-100, \mathrm{n}=47)$ \\
\hline
\end{tabular}

${ }^{a}$ n's represent actual number of patients included in each analysis. In cases where data were missing or incomplete, patients' data were not included;

BMI: Body-mass index.

Table 2: Preoperative and intraoperative opioid use.

\begin{tabular}{|l|l|l|}
\hline & Control $(\mathrm{n}=80)$ & SST $(\mathrm{n}=47)$ \\
\hline Patients Receiving Intraoperative IV Opioids & $97.5 \%$ & $61.7 \%^{+}$ \\
\hline Pre- and Intraoperative Total Opioid Dose (MME; Mean \pm SEM) & \\
\hline
\end{tabular}

ancludes MME of $5 \mathrm{mg}$ IV morphine in the SST group to account for preoperative SST dosing (Miner, et al.);

${ }^{+} p<0.001$ via Chi Square Test;

${ }^{\ddagger} p<0.001$ via Student's T Test;

MME: Morphine milligram equivalents; SEM: Standard error of the mean.

Table 3: Postoperative opioid use.

\begin{tabular}{|c|c|c|}
\hline & Control $(n=80)$ & SST $(n=47)$ \\
\hline Patients Requiring Postoperative Opioids & $63.0 \%$ & $10.6 \%^{+}$ \\
\hline Postoperative Total Opioid Dose(MME; mean \pm SEM) ${ }^{\mathrm{a}}$ & $4.4 \pm 0.5 \mathrm{mg}$ & $0.9 \pm 0.4 \mathrm{mg}^{\ddagger}$ \\
\hline
\end{tabular}

ancludes IV and oral opioids administered in the post-anesthesia care unit;

${ }^{\dagger} p<0.001$ via Chi Square Test;

${ }^{\ddagger} p<0.001$ via Student's T Test;

MME: Morphine milligram equivalents; SEM: Standard error of the mean.

the subject receiving the SST dose and incision was 35 minutes, with a range of 3-92 minutes. While $97.5 \%$ of control patients received IV opioids just prior to, or during, surgery, only $61.7 \%$ of SST-treated patients required intraoperative opioids following dosing with SST ( $p<0.001$; Table 2). In addition, the control patients received a total preoperative and intraoperative mean opioid dose of $20.0 \mathrm{mg} \mathrm{MME}$, while the mean dose for SST patients was $10.9 \mathrm{mg} \mathrm{MME}(p<0.001$; Table 2). Importantly, this total for the SST group consisted of $5.9 \mathrm{mg}$ MME from intraoperative IV opioid dosing and an additional preoperative $5.0 \mathrm{mg}$ MME from SST, which is the morphine-equivalent dose for SST $30 \mathrm{mcg}$ [16].

Similarly, while $63.0 \%$ of control patients required postoperative opioids, only $10.6 \%$ of SST patients required opioid dosing in the PACU ( $p<0.001$; Table 3$)$. The mean opioid requirement for the control group was $4.4 \mathrm{mg} \mathrm{MME}$, whereas the SST group was $0.9 \mathrm{mg} \mathrm{MME} \mathrm{(}<<0.001$; Table 3$)$.

Calculating the mean overall opioid dose throughout the outpatient surgical setting shows that control patients received a total of $24.6 \mathrm{mg} \mathrm{MME}$, while SST patients required less than half the opioid MME at $11.8 \mathrm{mg}(\mathrm{p}<0.001$; Figure 1$)$.
Table 4: Patients receiving supplemental IV medications.

\begin{tabular}{|l|l|l|}
\hline & Control $(n=80)$ & SST $(n=47)$ \\
\hline Adrenergic Agonist Use & $40.0 \%$ & $19 \%^{+}$ \\
\hline IV Acetaminophen & $90 \%$ & $38 \%^{\ddagger}$ \\
\hline Naloxone & $0 \%$ & $0 \%$ \\
\hline
\end{tabular}

aephedrine, norepinephrine, or phenylephrine;

${ }^{+} p=0.015$ via Chi Square Test;

${ }^{\ddagger} p<0.001$ via Chi Square Test.

Control patients were found to have a mean phase 1 PACU time of 54.9 minutes while their SST-treated counterparts had a mean PACU time of 36.3 minutes (Figure 2). The average PACU time for SST-treated patients was shown to be significantly lower than control patients by $34 \%(p<0.001)$.

Regarding supplemental IV medications, which were assessed as secondary endpoints, $40 \%$ of control patients required adrenergic agonists, such as ephedrine, for stabilization of heart rate or blood pressure intraoperatively, whereas $19 \%$ of SST patients required such treatment $(p=0.015$; Table 4). 


Figure 1: Overall morphine milligram equivalents utilized from preoperative to PACU discharge.
Data presented as mean \pm standard error of the mean;
f $p<0.001$ via Student's T Test;
MME: Morphine milligram equivalents.

Figure 2: Phase 1 postanesthesia care unit time.
Data presented as mean \pm standard error of the mean;
f $p<0.001$ via Student's T Test;
PACU: Postanesthesia care unit.

Ninety percent of control patients received IV acetaminophen $1000 \mathrm{mg}$ preoperatively, which was significantly reduced in the SST-treated group ( $38 \% ; \mathrm{p}<0.001)$ after early observations of decreased intra- and postoperative opioid requirements in the SST-treated patients were observed. No patients in either group required IV naloxone for respiratory depression.

In the SST group, there were no respiratory adverse events or any unexpected adverse reactions observed by the hospital staff.

\section{Discussion}

An increasing number of surgeries and procedures are being performed on an outpatient basis, largely due to the fact that over the past few decades, from laparoscopic cholecystectomies to knee arthroplasties, it has been shown that outpatient procedures are far less costly than the same pro- cedure performed in an inpatient setting $[17,18]$. This shift away from inpatient surgeries has been enabled because of improvements in anesthetic techniques, patient monitoring, and the development of minimally invasive surgical techniques. As the number of outpatient surgeries continues to rise, it becomes increasingly important to continue to leverage novel medical advances to enhance positive surgical outcomes and improve efficiency in ambulatory surgery settings.

In this medication use evaluation study, by administering a single preoperative dose of a sublingual opioid for acute pain management, we observed a reduction in intra- and postoperative opioid use, a reduced need for adrenergic agonists during surgery, and reduced time to discharge in the PACU. Preoperative administration of SST $30 \mathrm{mcg}$ significantly reduced opioid use relative to controls despite the fact that a larger percentage of control patients received preoperative IV acetaminophen. This finding was consistent when opioid 
Citation: Tvetenstrand CD, Wolff ME (2020) Reduced Opioid Use and Reduced Time in the Postanesthesia Care Unit Following Preoperative Administration of Sublingual Sufentanil in an Ambulatory Surgery Setting. J Clin Anesth Pain Manag 4(2):123-128

utilization was evaluated intraoperatively, postoperatively, as well as for overall use throughout the perioperative period. These observations are supported by published pharmacokinetic and pharmacodynamic data showing that SST provides sufficient sufentanil plasma concentrations to provide effective pain relief for up to 3 hours with a single $30 \mathrm{mcg}$ dose $[13,14]$ - which, when administered preoperatively in an ambulatory surgery setting, can provide analgesia throughout both the intraoperative and postoperative setting as shown in this study. This is in marked contrast to the pharmacokinetics and pharmacodynamics of IV bolus fentanyl administration that is commonly used in the ambulatory surgery setting. The initial rapid distribution half-life of IV fentanyl results in a short analgesic duration, with pain scores returning back to baseline within 30 minutes in patients recovering from ambulatory surgery $[6,19]$.

Intraoperatively, along with reduced IV opioid requirements, it was found that $50 \%$ fewer patients in the SST group required adrenergic agonists compared with controls. Since the sublingual administration of SST results in slower absorption and lower peak plasma levels relative to IV bolus administration of opioids [13], it is possible that SST resulted in greater intraoperative hemodynamic stability relative to IV bolus opioid administration, which is known to produce rapid high peak plasma concentrations, contributing to bradycardia and hypotension [5,7].

An important finding of this study was the $34 \%$ reduction in Phase 1 unit time for SST-treated patients compared to controls. Although respiratory depression and other serious adverse events were not observed during the conduct of the study in either group, we hypothesize that the reduced use of IV opioids during the procedure and in the PACU resulted in less postoperative sedation and other opioid-induced side effects, any of which could delay discharge from the PACU. Although ease of emergence from anesthesia was not a prespecified endpoint in this study, the PACU staff routinely stated that SST-treated patients woke up 'smoothly' and 'without confusion or agitation'. These observations are consistent with a recently published study that was performed in the emergency department that showed that $97 \%$ of SST-treated patients had no evidence of cognitive impairment as measured by the Six-item Screener [16].

This study is limited by the fact that it was open-label and was partially based on a retrospective analysis of a control patient population. In addition, the degree to which patients were opioid naïve or opioid tolerant was not controlled, which has the potential to affect the amount of opioid, required to provide sufficient analgesia. However, the baseline demographics and types of surgeries performed for the SST-treated and control groups were nearly identical and the fact that the surgeries were performed by the same surgeon, at the same facility, over a very similar timeframe within several months, suggests that these data can provide important information about the real-world use of SST in an ambulatory surgery setting. Finally, outside of the required preoperative dosing of SST in the prospective group, none of the other medications reported, such as opioids and adrenergic agents, were limited by the protocol and were administered only as needed by the anesthesiologist and PACU staff as documented in electronic medical records, thus providing objective evaluation of the prespecified endpoints. As this study evaluated abdominal surgery patients mainly, additional studies evaluating SST in other surgical specialties, painful procedures, burn dressing changes or emergency trauma settings would be beneficial.

\section{Conclusion}

In summary, these data show that preoperative administration of SST results in significant reductions in opioid requirements during outpatient surgery and shorter PACU stays. While adding a novel opioid to a hospital formulary seems counterintuitive with the constant legal and regulatory pressure to reduce opioid use, these observations strongly support utilizing SST over intravenous bolus opioids in alignment with opioid stewardship programs and enhanced recovery after surgery protocols, which focus on minimizing opioid dosing in multimodal analgesic protocols. Ultimately, the use of preoperative SST may result in a new paradigm for pain management protocols in the ambulatory surgery setting, which could provide significant cost savings due to less medical intervention and improved patient recovery.

\section{Funding}

Funding was not provided for the conduct of this study.

\section{Acknowledgements}

Medical writing support (funded by AcelRx Pharmaceuticals, Inc.) was provided by Eric R Kinzler, PhD and Gerald E Dodson, PhD of Neura Therapeutik who, on the behalf of the authors, developed the first draft based on an author-approved outline and assisted in implementing author revisions throughout the editorial process.

\section{References}

1. MI Gold, DJ Sacks, DB Grosnoff, et al. (1989) Use of esmolol during anesthesia to treat tachycardia and hypertension. Anesth Analg 68: 101-104.

2. DJ Pavlin, SE Rapp, NL Polissar, et al. (1998) Factors affecting discharge time in adult outpatients. Anesth Analg 87: 816-826.

3. Mather LE (1983) Clinical pharmacokinetics of fentanyl and its newer derivatives. Clin Pharmacokinet 8: 422-446.

4. Peng PW, AN Sandler (1999) A review of the use of fentanyl analgesia in the management of acute pain in adults. Anesthesiology 90: 576-599.

5. I Michelsen, HS Helbo-Hansen, F Køhler, et al. (1998) Prophylactic ephedrine attenuates the hemodynamic response to propofol in elderly female patients. Anesth Analg 86: 477-481.

6. SL Shafer, JR Varvel, N Aziz, et al. (1990) Pharmacokinetics of fentanyl administered by computer-controlled infusion pump. Anesthesiology 73: 1091-1102.

7. Egan TD (2019) Are opioids indispensable for general anaesthesia? Br J Anaesth 122: e127-e135.

8. Pavlin JD, CD Kent (2008) Recovery after ambulatory anesthesia. Curr Opin Anaesthesiol 21: 729-735.

9. Wheeler M, Gary M Oderda, Michael A Ashburn, et al. (2002) Adverse events associated with postoperative opioid analgesia: 
Citation: Tvetenstrand CD, Wolff ME (2020) Reduced Opioid Use and Reduced Time in the Postanesthesia Care Unit Following Preoperative Administration of Sublingual Sufentanil in an Ambulatory Surgery Setting. J Clin Anesth Pain Manag 4(2):123-128

A systematic review. J Pain 3: 159-180.

10. Iwata Y, Yoko Mizota, Toshiyuki Mizota, et al. (2012) Postoperative continuous intravenous infusion of fentanyl is associated with the development of orthostatic intolerance and delayed ambulation in patients after gynecologic laparoscopic surgery. J Anesth 26: 503-508.

11. MM Cohen, LL O’Brien-Pallas, C Copplestone, et al. (1999) Nursing workload associated with adverse events in the postanesthesia care unit. Anesthesiology 91: 1882-1890.

12. Bernards CM (1999) Clinical implications of physiochemical properties of opioids. In: C Stein, Opioids in Pain Control. (edn), Cambridge University Press, Cambridge, UK, 166-187.

13. Fisher DM, Peter Chang, D Russell Wada, et al. (2018) Pharmacokinetic properties of a sufentanil sublingual tablet intended to treat acute pain. Anesthesiology 128: 943-952.
14. Minkowitz HS, David Leiman, Timothy Melson, et al. (2017) Sufentanil sublingual tablet $30 \mathrm{mcg}$ for the management of pain following abdominal surgery: A randomized, placebo-controlled, phase-3 study. Pain Pract 17: 848-858.

15. (2020) Opioid calculator. Practical Pain Management.

16. Miner JR, Zubaid Rafique, Harold S Minkowitz, et al. (2018) Sufentanil sublingual tablet $30 \mathrm{mcg}$ for moderate-to-severe acute pain in the ED. Am J Emerg Med 36: 954-961.

17. Fleisher LA, K Yee, KD Lillemoe, et al. (1999) Is outpatient laparoscopic cholecystectomy safe and cost-effective? A model to study transition of care. Anesthesiology 90: 1746-1755.

18. Richter DL, DR Diduch (2017) Cost comparison of outpatient versus inpatient unicompartmental knee arthroplasty. Orthop J Sports Med 5.

19. Claxton AR, G McGuire, F Chung, et al. (1997) Evaluation of morphine versus fentanyl for postoperative analgesia after ambulatory surgical procedures. Anesth Analg 84: 509-514. 